\title{
Protein kinase D1 regulates hypoxic metabolism through HIF-1 $\alpha$ and glycolytic enzymes incancer cells
}

\author{
JIAO CHEN ${ }^{*}$, BOMIAO CUI $^{*}$, YAPING FAN ${ }^{*}$, XIAOYING LI, QIAN LI, YUE DU, YUN FENG and PING ZHANG \\ State Key Laboratory of Oral Diseases, West China Hospital of Stomatology, \\ Sichuan University, Chengdu, Sichuan 610041, P.R. China
}

Received October 7, 2017; Accepted April 3, 2018

DOI: $10.3892 /$ or.2018.6479

\begin{abstract}
Protein kinase D1 (PKD1), one of the protein kinase D (PKD) family members, plays a prominent role in multiple bio-behaviors of cancer cells. Low $\mathrm{pH}$ and hypoxia are unique characteristics of the tumor microenvironment. The aim of this study was to investigate the role and mechanism of PKD1 in regulating metabolism in the human tongue squamous cell carcinoma (TSCC) cell line SCC25 under a hypoxic condition, as well as growth and apoptosis. Here, we found that hypoxia not only induced the expression of HIF-1 $\alpha$, but also induced the expression and activation of PKD1. Moreover, we inhibited the expression of PKD1 by shRNA interference, and the growth of SCC25 cells under hypoxia was significantly decreased, as well as the expression of HIF-1 $\alpha$, while the percentage of apoptotic SCC25 cells was increased. Furthermore, stable silencing of PKD1 in SCC25 cells under a hypoxic condition decreased glucose uptake, lactate production and glycolytic enzyme (GLUT-1 and LDHA) expression, as well as reduced the phosphorylation of p38 MAPK. The results revealed that following inhibition of the expression of PKD1 under a hypoxic condition, the growth and metabolism of the SCC25 cells were significantly suppressed. In contrast, when PKD1 was overexpressed in SCC25 cells, the results were completely reversed, except for growth and apoptosis. Taken together, our results demonstrated that PKD1 not only regulates the hypoxic glycolytic metabolism of cancer cells via regulation of the expression of HIF-1 $\alpha$ and glycolytic enzymes, but is also involved in the remodeling of the acidic tumor microenvironment. This study suggests that PKD1 may be a potential target for microenvironment-directed tumor biotherapy.
\end{abstract}

Correspondence to: Professor Yun Feng or Professor Ping Zhang, State Key Laboratory of Oral Diseases, West China School of Stomatology, Sichuan University, 14 Renmin South Road, Section 3, Chengdu, Sichuan 610041, P.R. China

E-mail: 953463551@qq.com

E-mail: pingzhang68@hotmail.com

*Contributed equally

Key words: PKD1, hypoxia, SCC25, HIF-1 $\alpha$, glycolytic enzymes

\section{Introduction}

Oral cancer is a major public health issue and a social challenge. Tongue squamous cell carcinoma (TSCC) is the most aggressive type of oral cancer. Environmental factors, genetics and immune status have been confirmed to be related to the tumorigenesis of tongue cancer (1). The tumor microenvironment, which is composed of complex components including stem cells, fibroblasts, immune cells and their secreted factors, is critical to the initiation, development and maintenance of tumorigenesis (1). As the 'residence niche' of cancer cells, the composition and structure of the tumor microenvironment not only affects the bio-behavior, but also determines the survival and elimination of tumor cells (1-3). Targeting the tumor microenvironment by remodeling its components and construction could be a useful a strategy to treat and prevent cancer (4).

Low $\mathrm{pH}$ and hypoxia are unique characteristics of the tumor microenvironment $(5,6)$. Glucose metabolism supplies energy for the growth and maintainence of mammalian cells (7), and cancer cells are well-known to employ glycolysis for energy metabolism $(8,9)$. As soon as the primary tumor outgrows its blood supply and creates a hypoxic microenvironment, cancer cells typically switch to a glycolytic metabolism and secrete lactic acid, creating a low $\mathrm{pH}$ microenvironment (10-14). An acidic and hypoxic microenvironment is not only toxic to the surrounding normal somatic cells, but also induces the expression of HIF-1 $\alpha$, inhibits antitumor immune responses and promotes proliferation, invasion, metastases and recurrence (12,15-17). Investigation and elucidation of the signaling mechanism of hypoxic metabolism of cancer cells is critical to explore new cancer treatment strategies (18).

Protein kinase D (PKD) is an evolutionarily conserved serine/threonine protein kinase with structural, enzymological and regulatory properties distinct from those of PKC (19). PKD belongs to the calmodulin-dependent protein kinase (CAMK) superfamily that also includes PKD1/PKC $\mu$, PKD2 and PKD3/PKCv (19,20). PKDs can be activated by a number of external stimuli, including G-protein-coupled receptor agonists, hormones, chemokines, bioactive lipids and growth factors $(19,21)$. PKDs mediate diverse and complex biological functions throughout the body, including cell proliferation and differentiation, signal transduction, membrane trafficking, secretion, immunoregulation and 
angiogenesis $(19,21,22)$. PKDs play important roles in tumor proliferation and differentiation, migration, apoptosis, epithelial-to-mesenchymal transition (EMT), angiogenesis, MDR, oxidative stress, autophagy and transcriptional regulation (20,23-25). PKD1, one of the most well-studied PKD family members, is expressed and activated to varying degrees in cancer cells from many different tissues of origin, and has distinct roles based on the tissue of origin $(19,24,26)$. It is overexpressed in pancreatic cancer and skin cancers. However, the expression of PKD1 is downregulated in prostate, breast and gastric cancer $(19,23,27,28)$. Abnormal expression of PKD1 is often associated with tumorigenesis, progression, apoptosis, invasion and poor prognosis $(19,25,29)$.

In the present study, we investigated the role of PKD1 in hypoxic glycolysis in SCC25 cancer cells. We found that hypoxia not only induced the expression of HIF-1 $\alpha$, but also promoted PKD1 expression and activation, through phosphorylation. PKD1 not only regulated the growth and apoptosis of SCC25 cells under hypoxic conditions, but also promoted glycolytic metabolism ofSCC25 cells by promoting the expression of HIF-1 $\alpha$, GLUT1, LDHA and p38 MAPK within a hypoxic microenvironment.

\section{Materials and methods}

Antibodies and reagents. Antibodies against PKD1 rabbit mAb (cat. no. 90039), phospho-PKD1 rabbit Ab (cat. no. 2051), p38 MAPK rabbit mAb (cat. no. 8690), phospho-p38 MAPK (Thr180/Tyr182) rabbit $\mathrm{mAb}$ (cat. no. 4511), LDHA rabbit $\mathrm{mAb}$ (cat. no. 3582) and $\beta$-actin rabbit $\mathrm{mAb}$ (cat. no. 8457) were obtained from Cell Signaling Technology, Inc. (Beverly, MA, USA). Antibodies against HIF-1 $\alpha$ rabbit mAb (cat. no. ab51608), GLUT-1 rabbit pAb (cat. no. ab51608) and LC3 rabbit pAb (cat. no. ab51520) were obtained from Abcam (Cambridge, MA, USA). Annexin V-FITC apoptosis detection and lactate assay kits were purchased from Sigma-Aldrich (Merck KGaA, Darmstadt, Germany). We purchased 2-NBDG [2-[N-(7-nitrobenz-2-oxa-1,3-diazol-4-yl)amino]-2-deoxyD-glucose] from Cayman Chemicals (Ann Arbor, MI, USA). Human PKD1 shRNA and control shRNA plasmids were obtained from Thermo Fisher Scientific, Inc. (Waltham, MA, USA). Human pEZ-Lv105-PKD1-overexpressing plasmid vectors were purchased from GeneCopoeia, Inc. (Rockville, MD, USA). The Cell Counting Kit (CCK-8) was obtained from Dojindo Molecular Technologies, Inc. (Kumamoto, Japan).

Cell culture and hypoxia treatment. Human oral squamous cell carcinoma (SCC25) cells were cultured in DME/F-12 (1:1) medium supplemented with $10 \%$ (vol/vol) fetal bovine serum (FBS), 1\% (vol/vol) penicillin/streptomycin mixture and $10 \mu \mathrm{M}$ hydrocortisone (both from Gibco, Thermo Fisher Scientific, Inc.). H1975, SCC25 and HSC-4 cells used in this study were purchased from the American Type Culture Collection (ATCC; Manassas, VA, USA) and cultured at the State Key Laboratory of Oral Diseases in a humidified atmosphere of $5 \% \mathrm{CO}_{2}$ at $37^{\circ} \mathrm{C}$. To induce hypoxia, SCC25 cells were placed in a chamber filled with a gas mixture of $1 \% \mathrm{O}_{2}$, $5 \% \mathrm{CO}_{2}$ and $94 \% \mathrm{~N}_{2}$.
Establishment of stable PKD1-knockdown and-overexpressing SCC25 cells. Human LVRH1GP-PKD1 shRNA (clone ID NM_002742.2) and LVRU6GP-control shRNA plasmids were obtained from Thermo Fisher Scientific, Inc. The PKD1-1-SH target sequences are as follows: gcaacaatat cccactcatga. PKD1-2-SH target sequences are as follows: gcaggtactacaaggaaattc. The shRNAs were transfected into the SCC25 cells using Lipofectamine 2000 reagent (Invitrogen; Thermo Fisher Scientific, Inc.) according to the manufacturer's protocol. Positive SCC25 clones were selected for at least two passages by using puromycin treatment at a concentration of $0.5 \mu \mathrm{g} / \mathrm{ml}$. After two weeks of growth in selective medium, the cells were harvested and the expression level of PKD1 was determined by western blot analysis. Stable PKD1-overexpressing SCC25 cells were generated similarly.

Cell viability assay. The CCK-8 was used to detect the cell viability of the SCC25 cell group (as the normal control group), the PKD1-knockdown group (PKD1-SH), PKD1-overexpressing group (PKD1-OE) and SCC25 control groups (Con-SH and Con-OE). SCC25 cells were seeded at a density of $1 \times 10^{3}$ cells/well in 96-well plates and cultured at $37^{\circ} \mathrm{C}$ in $1 \% \mathrm{O}_{2}, 5 \% \mathrm{CO}_{2}$ and $94 \% \mathrm{~N}_{2}$. Cell viability was assessed by measuring the absorbance of the converted dye at $450 \mathrm{~nm}$.

Annexin V-FITC apoptosis assay. SCC25 cells (wild-type SCC25, PKD1-SH, Con-SH, PKD1-OE and Con-OE) were seeded in 6-well plates atadensity of $2 \times 10^{5}$ cells/well and cultured at $37^{\circ} \mathrm{C}$ in $1 \% \mathrm{O}_{2}, 5 \% \mathrm{CO}_{2}$ and $94 \% \mathrm{~N}_{2}$. Annexin $\mathrm{V}$-fluorescein isothiocyanate (FITC) and propidium iodide (PI) for flow cytometry was used to identify the percentage of apoptotic cells following the manufacturer's protocol [Annexin V-FITC apoptosis detection kit (Sigma-Aldrich; Merck KGaA)]. Annexin $\mathrm{V}^{+} / \mathrm{PI}^{-}$apoptotic cells were identified via flow cytometry (FCM) using Summit 5.2 software (Beckman Coulter, Miami, FL, USA).

Glucose uptake assay. To analyze glucose uptake, the fluorescent glucose analog 2-NBDG was used for direct quantification of glucose incorporation in living cells by FCM (Beckman Coulter) (7,30). SCC25 cells were seeded in 6 -well plates and subjected to hypoxia $\left(1 \% \mathrm{O}_{2}, 5 \% \mathrm{CO}_{2}\right.$ and $94 \% \mathrm{~N}_{2}$ ). After treatment for $24 \mathrm{~h}$, the medium was removed and new medium containing $10 \mu \mathrm{M} 2-\mathrm{NBDG}$ was added. The cells were then incubated at $37^{\circ} \mathrm{C}$ in $1 \% \mathrm{O}_{2}, 5 \% \mathrm{CO}_{2}$ and $94 \% \mathrm{~N}_{2}$ for $30 \mathrm{~min}$. Cells were washed twice with Dulbecco's phosphate-buffered saline (DPBS; Invitrogen; Thermo Fisher Scientific, Inc.) then trypsinized, centrifuged 1,200 rpm for 10 min and resuspended in DPBS. A flow cytometer was used to analyze the uptake of 2-NBDG in the different cell groups.

Lactate assay. SCC25 cells were seeded in 6-well plates at a density of $2 \times 10^{5}$ cells/well and cultured at $37^{\circ} \mathrm{C}$ in $1 \% \mathrm{O}_{2}$, $5 \% \mathrm{CO}_{2}$ and $94 \% \mathrm{~N}_{2}$ for $24 \mathrm{~h}$. Lactate in the medium was measured using a lactate assay kit according to the manufacturer's instructions (Sigma-Aldrich; Merck KGaA).

Western blot analysis. Cells were collected and protein was collected using a Protein Extraction kit (Sigma-Aldrich; 
$\mathbf{A}$

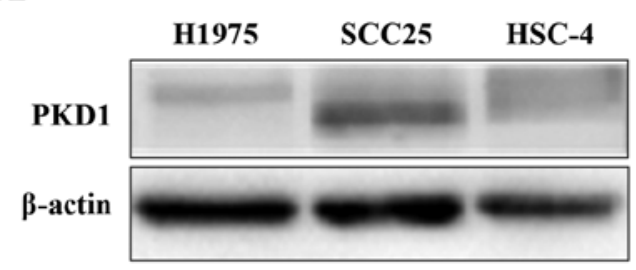

C

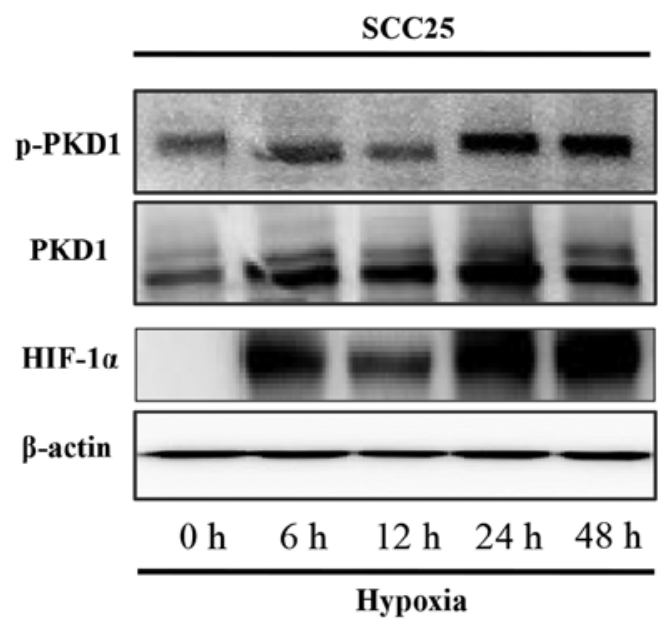

B

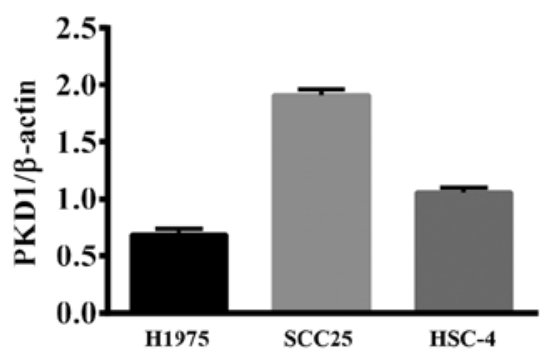

D
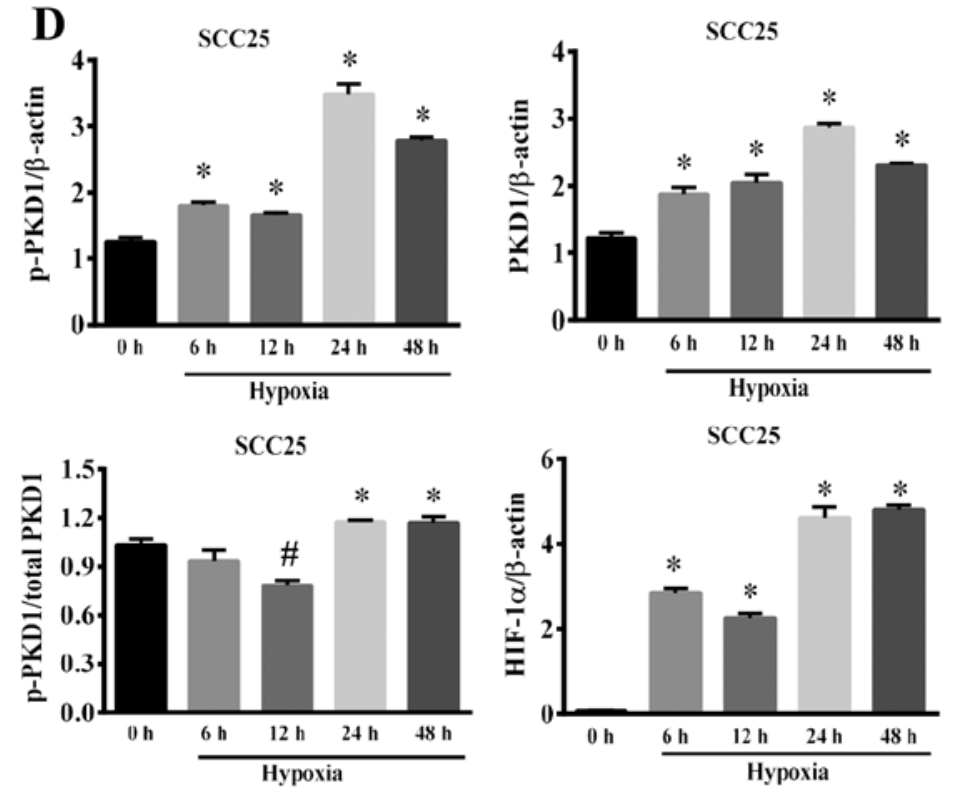

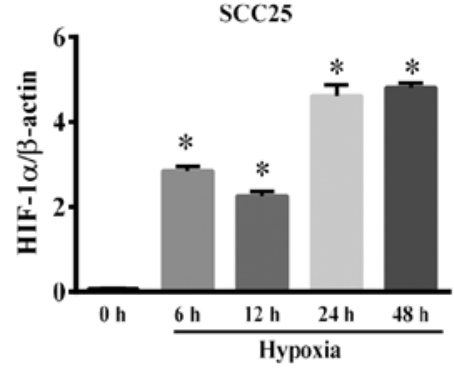

Figure 1. Hypoxia induces PKD1 and HIF-1 $\alpha$ expression in SCC25 cancer cells. SCC25 cells were cultured in a humidified atmosphere of $5 \% \mathrm{CO}_{2}, 1 \% \mathrm{O}_{2}$ and $94 \% \mathrm{~N}_{2}$ at $37^{\circ} \mathrm{C}$. Cells were collected at different times. The expression of p-PKD1, PKD1 and HIF-1 $\alpha$ were detected by western blot analysis. (A) Western blot analyses for PKD1 expression in H1975, SCC25 and HSC-4 cancer cells. (B) Quantification of PKD1 by densitometry analysis. (C) The expression of p-PKD1, PKD1 and HIF-1 $\alpha$ following different periods of exposure to hypoxia $\left(1 \% \mathrm{O}_{2}\right)$. Images are representative of three independent experiments. (D) Quantification of PKD1 phosphorylation (left upper panel) and total PKD1 (right upper panel), the ratio of p-PKD1/total PKD1 (left lower panel) and quantification of HIF-1 $\alpha$ (right lower panel) by densitometry analysis. Data are presented as the mean \pm SD. ${ }^{*} \mathrm{P}<0.05$ and ${ }^{\#} \mathrm{P}<0.05 \mathrm{vs} .0 \mathrm{~h}$.

Merck KGaA). The protein concentration was measured using a BCA protein assay kit (Thermo Fisher Scientific, Inc.). Total protein $(20 \mu \mathrm{g})$ was separated using 6 or $10 \%$ SDS-PAGE and transferred onto PVDF membranes (Bio-Rad Laboratories, Hercules, CA, USA). The membranes were blocked with 5\% skim milk and incubated with primary antibodies against PKD1, phospho-PKD1, HIF-1 $\alpha$, LC3, GLUT-1, LDHA, phosphop38 MAPK, p38 MAPK or $\beta$-actin at a dilution of 1:1,000 at $4^{\circ} \mathrm{C}$ overnight. Membranes were washed with TBST and then incubated with horseradish peroxidase (HRP)-conjugated secondary antibodies at a dilution of 1:2,000 for $2 \mathrm{~h}$ at room temperature. Luminescent signals were detected by ECL Western Blotting Substrate (Millipore, Billerica, MA, USA). Band intensity was quantified using Quantity One Software (Bio-Rad Laboratories). Phospho-p38 MAPK/p38 MAPK was defined as the phosphorylated index (PI) of p38 MAPK.

Statistical analysis. All data are presented as mean \pm standard deviation (SD). Significant differences between groups were analyzed by one-way analysis of variance (ANOVA), followed by a Bonferroni's post hoc test. A P-value $<0.05$ was considered to indicate a statistically significant difference. Graphs were plotted and analyses were performed using GraphPad Prism 7 software (GraphPad Software, Inc., La Jolla, CA, USA).

\section{Results}

Hypoxia induces PKD1 expression in SCC25 cancer cells. The SCC25 cell line is the most common type of human oral squamous cell carcinoma. In our pre-experiment, we investigated the expression of PKD1 in H1975, SCC25 and HSC-4 cancer cell lines. The results showed that PKD1 was expressed in SCC25 cells at a high level (Fig. 1A and B); therefore, we selected SCC25 as the target cell line for further study.

Hypoxia is a common characteristic of the tumor microenvironment. Hypoxia induces cancer cells to express HIF-1 $\alpha$ to initiate the expression of tumor growth factor and provide a growth signal for cancer cells. On the other hand, hypoxia promotes cancer cell switch to glycolysis for energy generation. To determine the role of PKD1 in regulating glycolytic metabolic progression of tumor cells, we first analyzed the expression and activation of PKD1 in tumor cells under a hypoxic microenvironment. SCC25 cells were cultured in a humidified atmosphere of $5 \% \mathrm{CO}_{2}, 1 \% \mathrm{O}_{2}$ and $94 \% \mathrm{~N}_{2}$ at $37^{\circ} \mathrm{C}$ to simulate a hypoxic tumor microenvironment. The expression of PKD1, p-PKD1 and HIF-1 $\alpha$ were detected by western blot analysis. As shown in Fig. 1C, SCC25 cells exposed to $6 \mathrm{~h}$ of hypoxia exhibited enhanced expression of p-PKD1, PKD1 and HIF-1 $\alpha$. In particular, when SCC25 cells were subjected 
A
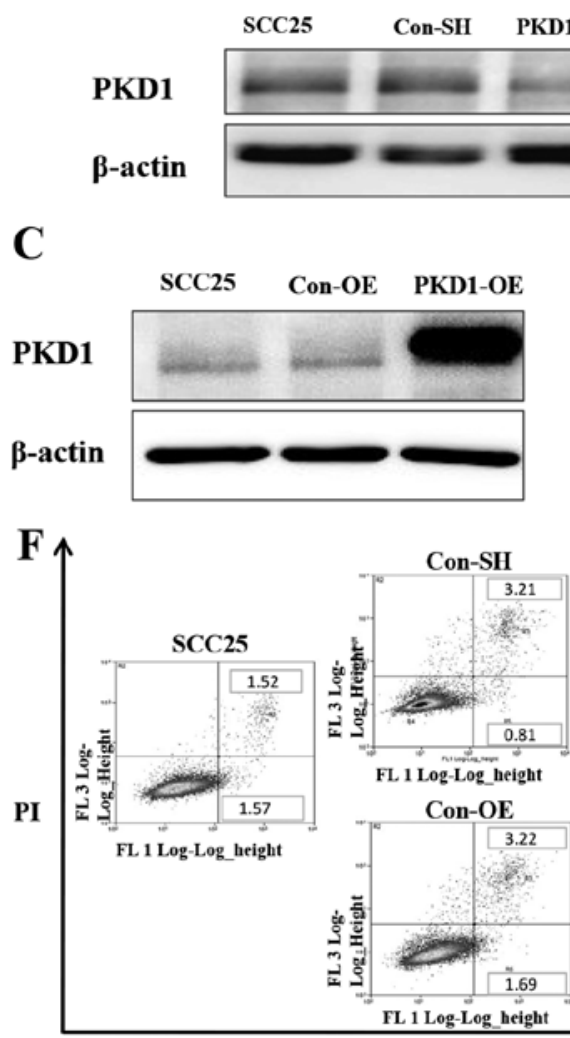

Annexin-FITC
D
B
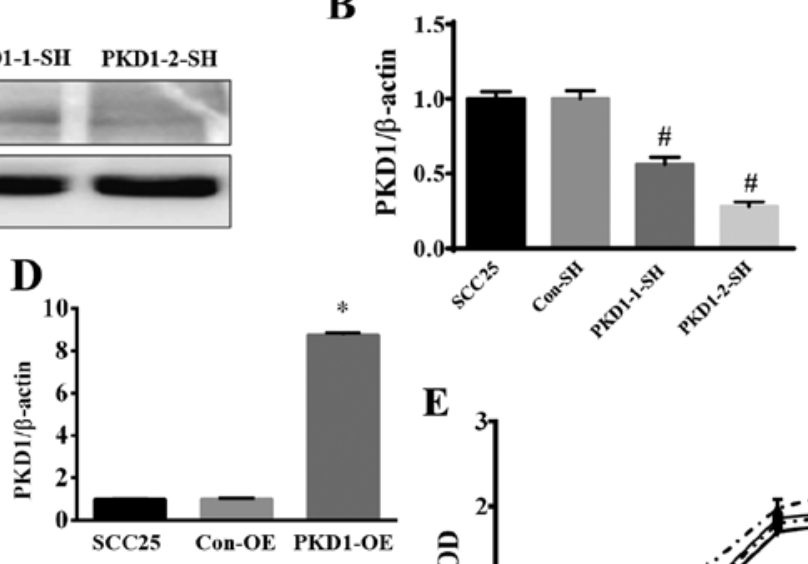

PKD1-SH

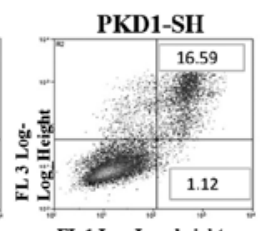

PKD1-OE
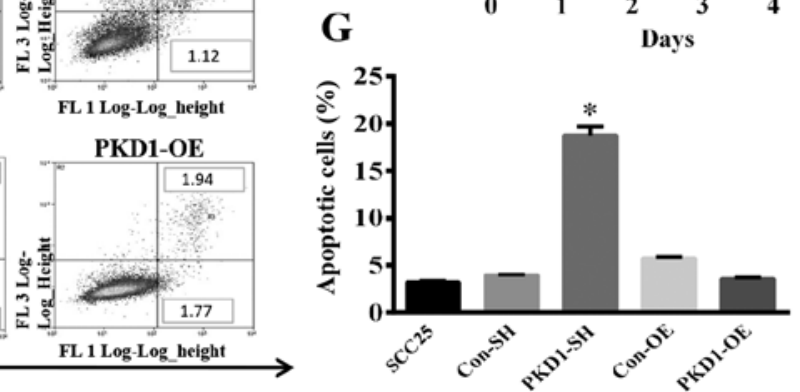

$\mathbf{E}$

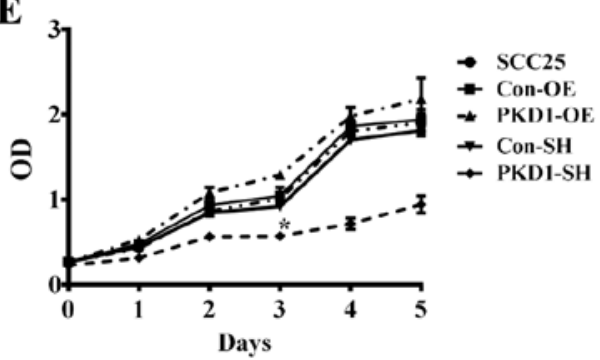

Figure 2. PKD1 regulates the growth and apoptosis of SCC25 cancer cells under hypoxia. SCC25 cells were transfected with the PKD1-shRNA plasmid to knockdown PKD1 expression (PKD1-SH) or the PKD1 pCDNA3.1/NT-PKD1 plasmid to overexpress PKD1 (PKD1-OE). (A) The expression of PKD1 in SCC25 cells after transfection with PKD1-shRNA (PKD1-SH) or control shRNA plasmid (Con-SH) as analyzed by western blot analysis. (B) Quantification of PKD1 by densitometry analysis after transfection with the shRNA plasmid. (C) The expression of PKD1 in SCC25 cells after transfection with pCDNA3.1/NT-PKD1(PKD1-OE) or control plasmid (Con-OE) as analyzed by western blot analysis. (D) Quantification of PKD1 by densitometry analysis after transfection with the pEZ-Lv105-PKD1 plasmid. (E) Growth curves of SCC25 cells after PKD1 knockdown or overexpression under hypoxia. (F) The apoptosis of SCC25 cells was evaluated using flow cytometry. (G) Quantification of the percentage of apoptotic cells. Images are representative of three independent experiments. Data are presented as the mean $\pm \mathrm{SD}$. ${ }^{*} \mathrm{P}<0.05$, significantly increased as compared to the control or wild-type $\mathrm{SCC} 25$ cells. ${ }^{*} \mathrm{P}<0.05$, significantly reduced as compared to the control.

to $24 \mathrm{~h}$ of hypoxia, the expression of p-PKD1, PKD1 and HIF-1 $\alpha$ reached the highest level (Fig. 1D). Moreover, the ratio of p-PKD1/PKD1 was significantly increased (Fig. 1D, left lower panel). Based on the above-mentioned research results, treatment with $24 \mathrm{~h}$ hypoxia was selected for the subsequent experiments. This result indicates that hypoxia not only induces the expression of PKD1, but also induces the phosphorylation and activation of PKD1.

PKDI promotes the growth and inhibits the apoptosis of SCC25 cells in a hypoxic microenvironment. Toleration of a hypoxic microenvironment is a characteristic of tumor cells. To investigate the role of PKD1 in the growth and apoptosis of tumor cells grown under hypoxia, SCC25 cells were transfected with PKD1 shRNA (PKD1-1-SH and PKD1-2-SH) to knockdown PKD1. Compared with the negative control (Con-SH), the expression of PKD1 was significantly reduced, as shown by western blot analysis (Fig. 2A and B). As shown in Fig. 2A and B, PKD1-2-SH was found to be the most effective shRNA plasmid with which to knockdown PKD1. Thus, we used PKD1-2-SH to knockdown PKD1 in the following experiments. Additionally, SCC25 cells were transfected with a pCDNA3.1/NT-PKD1 plasmid with a pre-inserted PKD1 gene for PKD1 overexpression (PKD1-OE) (Fig. 2C and D). PKD1-SH, PKD1-OE and wild-type SCC25 cells were cultured in a humidified atmosphere of $1 \% \mathrm{O}_{2}, 5 \% \mathrm{CO}_{2}$ and $94 \% \mathrm{~N}_{2}$ at $37^{\circ} \mathrm{C}$. Live cells were analyzed using the CCK-8 cell counting kit; apoptotic cells were analyzed via FCM. As shown in Fig. 2E, the cell growth curve demonstrated a significantly highest cell death at 3 days in the PKD1-SH group when compared with the other 4 tested groups. Importantly, SCC25 cell apoptosis was significantly increased after PKD1 knockdown with PKD1-SH (Fig. 2F and G). However, the results revealed that there was no significant difference in growth between the PKD1-Con and PKD1-OE group, and growth of the PKD1-overexpressing cells was not markedly affected according to the cell growth curve (Fig. 2E). These results indicate that PKD1 plays an important role in maintaining the survival and growth of SCC25 cells in a hypoxic microenvironment.

PKD1 regulates HIF-1 $\alpha$ expression. PKD1 is a protease kinase that is involved in the bio-behavior of cancer cells. HIF-1 $\alpha$ is an important regulator of the glucose metabolism in cancer cells. To reveal the role of PKD1 in the progression of hypoxia-induced HIF- $1 \alpha$ expression, wild-type SCC25, 
A

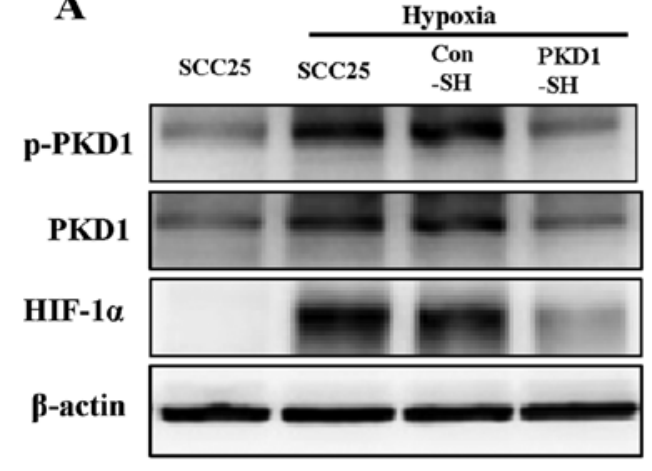

C
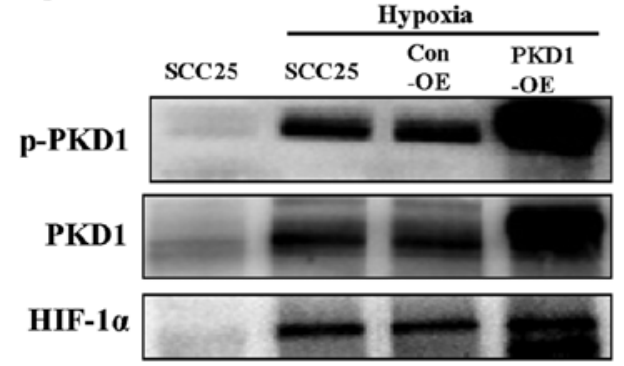

B-actin

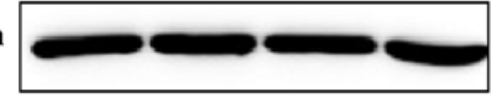

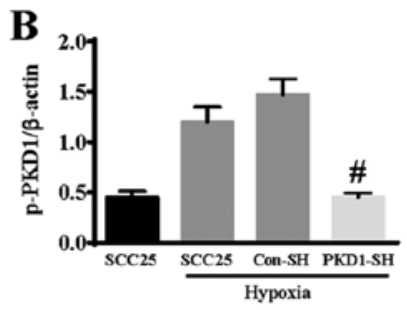
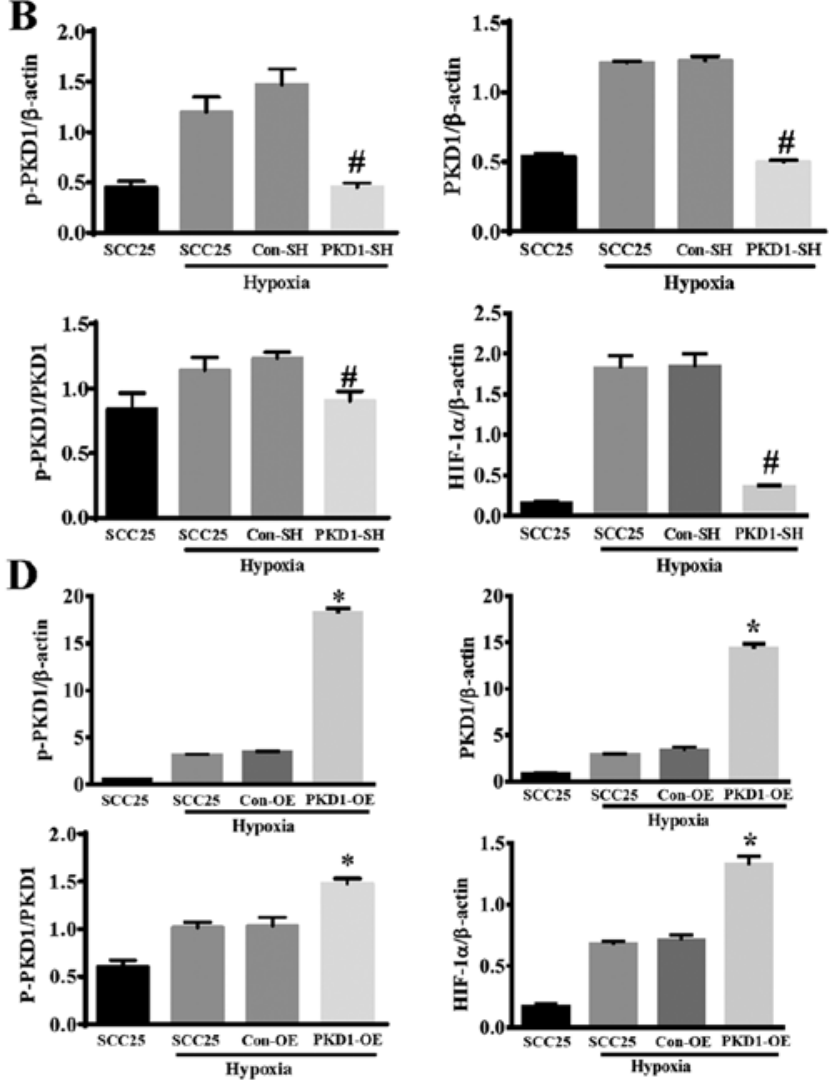
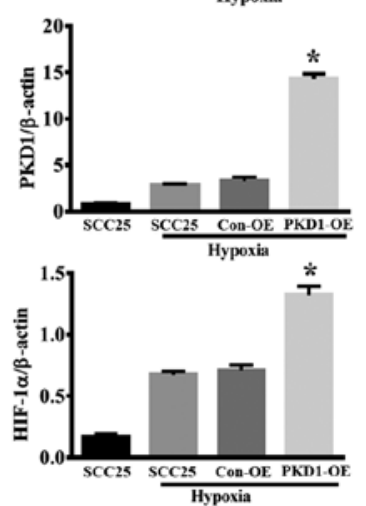

Figure 3. PKD1 promotes hypoxia-induced HIF-1 $\alpha$ expression. PKD1-SH and PKD1-OE SCC25 cells were cultured in a humidified atmosphere of 5\% CO $1 \% \mathrm{O}_{2}$ and $94 \% \mathrm{~N}_{2}$ at $37^{\circ} \mathrm{C}$. The expression of PKD1, p-PKD1 and HIF-1 $\alpha$ were detected by western blot analysis. (A) The expression of PKD1, p-PKD1 and HIF-1 $\alpha$ in PKD1-SHSCC25 cells under hypoxia. (B) Quantification of PKD1 phosphorylation (left upper panel) and total PKD1 (right upper panel), the ratio of p-PKD1/total PKD1 (left lower panel) and quantification of HIF-1 $\alpha$ (right lower panel) by densitometry analysis. (C) The expression of PKD1, p-PKD1 and HIF-1 $\alpha$ in PKD1-OE SCC25 cells under hypoxia. (D) Quantification of PKD1 phosphorylation (left upper panel) and total PKD1 (right upper panel), the ratio of p-PKD1/total PKD1 (left lower panel) and quantification of HIF-1 $\alpha$ (right lower panel) by densitometry analysis. Images are representative of three independent experiments. Data are presented as the mean \pm SD. ${ }^{*} \mathrm{P}<0.05$, significantly increased as compared to the control plasmid or wild-type SCC 25 cells under hypoxia; ${ }^{\#} \mathrm{P}<0.05$, significantly reduced as compared to the control plasmid or wild-type SCC25 cells under hypoxia.

PKD1-SH and PKD1-OESCC25 cells were cultured in a humidified atmosphere of $5 \% \mathrm{CO}_{2}, 1 \% \mathrm{O}_{2}$ and $94 \% \mathrm{~N}_{2}$ at $37^{\circ} \mathrm{C}$ for $24 \mathrm{~h}$. SCC25 cells cultured in normal condition as a control, PKD1-knockdown or -overexpressing SCC25 cells did not exhibit a significant difference in the expression of HIF-1 $\alpha$ under a normal condition (data not shown). PKD1 and HIF-1 $\alpha$ expression was evaluated by western blot analysis. As shown in Fig. 3, the phosphorylation and activation of PKD1 and HIF-1 $\alpha$ expression was significantly increased in the SCC25 cells under hypoxia. Moreover, the expression of HIF-1 $\alpha$ was significantly inhibited after PKD1 knockdown by PKD1-SH (Fig. 3A and B). On the other hand, overexpression of PKD1 and phosphorylated activation significantly promoted hypoxia-induced HIF-1 $\alpha$ expression (Fig. 3C and D). These results indicate that $\mathrm{PKD} 1$ is an important regulator of hypoxia-induced HIF-1 $\alpha$ expression.

PKD1 promotes glycolysis progression in SCC 25 cells. The Warburg effect is a unique characteristic of cancer cell glycometabolism. Cancer cells uptake glucose and release lactate through glycolysis under hypoxic conditions. Detection of the ability of cancer cells to uptake glucose and release lactate in culture media can reveal the glycolytic activity of cancer cells. Wild-type SCC25, PKD1-SH and PKD1-OESCC25 cells were cultured in medium with $10 \mathrm{nM}$ 2-NBDG for $30 \mathrm{~min}$. Glucose uptake and lactate release by cancer cells were analyzed via FCM and lactate assay kits, respectively. As shown in Fig. 4A and B, hypoxia stimulated SCC25 cells to uptake glucose and release lactate. When PKD1 was knocked down with PKD1-SH, the ability of SCC25 cells to uptake glucose was significantly reduced (Fig. 4A, left panel), as lactate release was also reduced (Fig. 4B, left panel). Conversely, when PKD1 was overexpressed, the ability of SCC 25 cells to uptake glucose and release lactate was significantly increased (Fig. 4A and B, right panels). These results indicate that PKD1 is an important signaling molecule promoting cancer cell glycolysis.

We also detected the growth and the expression of autophagy proteins by SCC25 cells cultured under an acidic environment after knockdown of PKD1. As shown in Fig. 4C, the growth of SCC25 cells was significantly inhibited under an acidic condition. Fig. 4D shows that the expression of autophagy proteins was significantly decreased (LC3-II/LC-3I). These data indicated that PKD1 moderated autophagy to promote the adaptation of tumor cells to an acidic microenvironment.

PKD1 promotes cancer cel lglycolysis by promoting GLUT1 andLDHA expression. GLUT1 and LDHA are two key enzymes involved in the process of glycolysis. Hypoxia induced both 
A

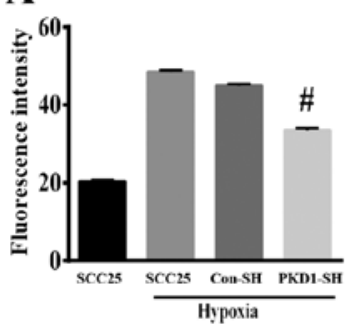

C

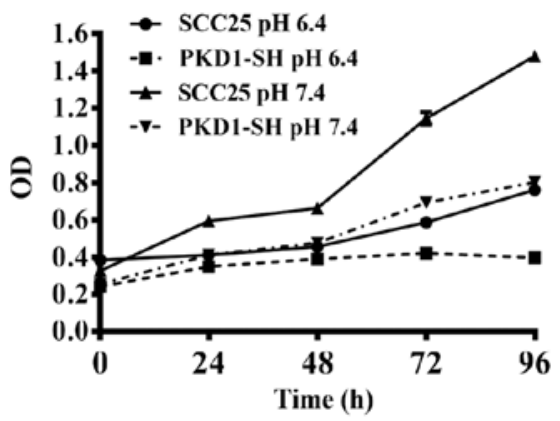

B
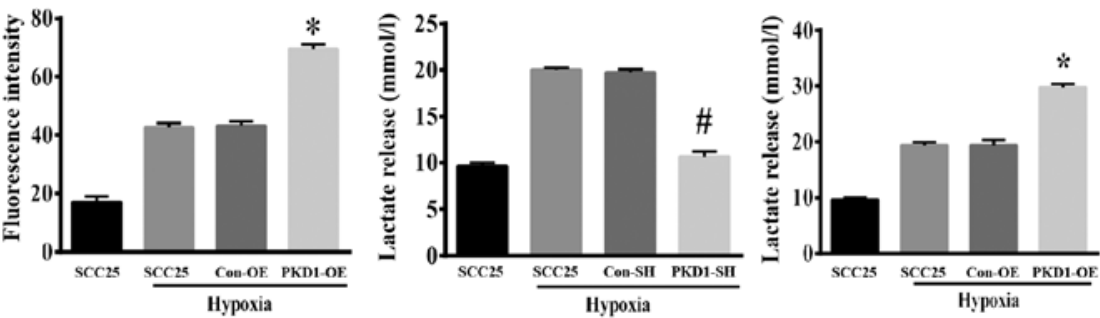

D
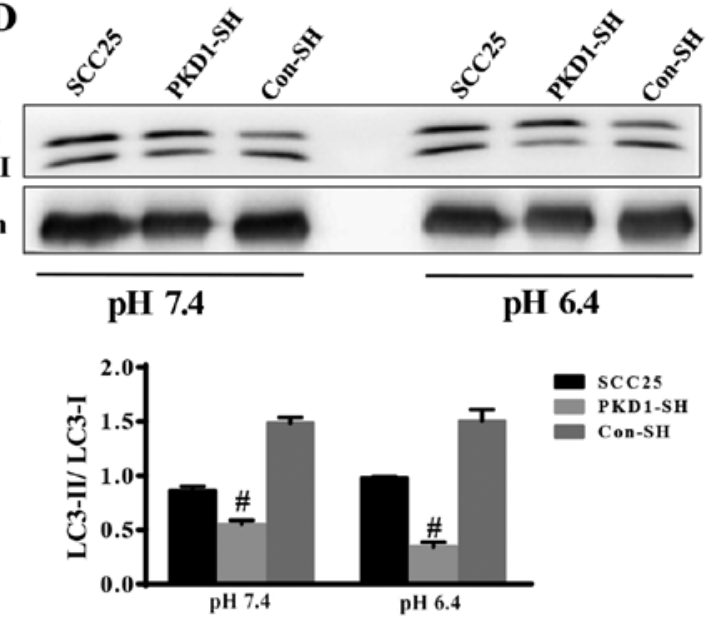

Figure 4. PKD1 promotes glucose uptake and lactate release in SCC25 cells under hypoxia. PKD1-SH and PKD1-OE SCC25 cells were cultured in a humidified atmosphere of $5 \% \mathrm{CO}_{2}, 1 \% \mathrm{O}_{2}$ and $94 \% \mathrm{~N}_{2}$ at $37^{\circ} \mathrm{C}$. Glucose uptake and lactate release were detected via flow cytometry and a lactate assay kit, respectively. (A) Quantification of 2-NBDG uptake assays after PKD1 knockdown (left panel). Quantification of 2-NBDG uptake assays after PKD1 overexpression (right panel). (B) Quantification of lactate release after PKD1 knockdown (left panel). Quantification of lactate release after PKD1 overexpression (right panel). (C) Growth curves of SCC25 cells under an acidic condition. (D) The expression of autophagy protein (LC3-II/LC-3I) as detected by western blot analysis (upper panel). Bar graph shows the quantification of LC3-II/LC-3I (lower panel). Data are presented as the mean \pm SD. *P $<0.05$, significantly increased as compared to the control plasmid or wild-type SCC 25 cells under hypoxia; ${ }^{\#} \mathrm{P}<0.05$, significantly reduced as compared to the control plasmid or wild-type SCC25 cells under hypoxia.

A

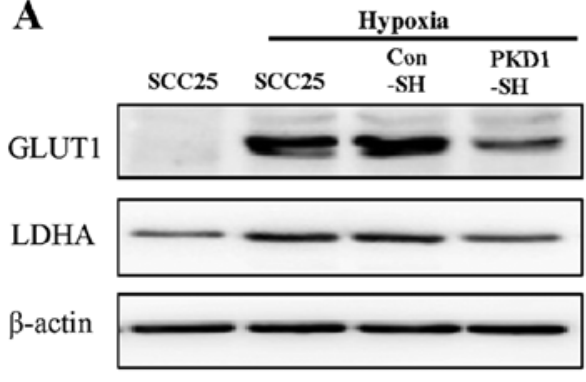

C

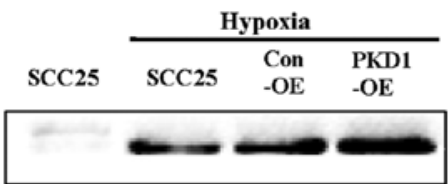

LDHA

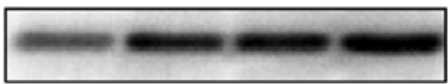

$\beta$-actin

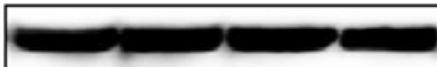

B
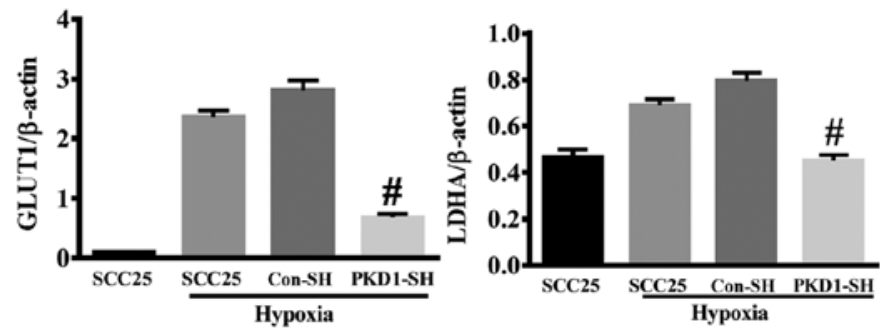

D

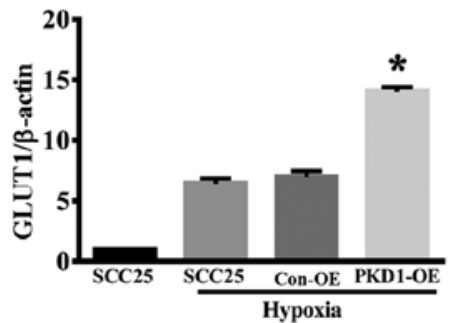

Figure 5. PKD1 promotes the expression of glycolytic enzymes under hypoxia. PKD1-SH and PKD1-OE SCC25 cells were cultured in a humidified atmosphere of 5\% $\mathrm{CO}_{2}, 1 \% \mathrm{O}_{2}$ and $94 \% \mathrm{~N}_{2}$ at $37^{\circ} \mathrm{C}$. (A) Expression of GLUT1 and LDHA after PKD1 knockdown as detected by western blot analysis. (B) Quantification of GLUT1 (left panel) and LDHA (right panel) after PKD1 knockdown. (C) Expression of GLUT1 and LDHA after PKD1 overexpression as detected by western blot analysis. (D) Quantification of GLUT1 (left panel) and LDHA (right panel) after PKD1 overexpression. Figures are representative of three independent experiments. Data are presented as the mean $\pm \mathrm{SD}$. "P<0.05, significantly increased as compared to control plasmid or wild-type SCC25 cells under hypoxia; ${ }^{\#} \mathrm{P}<0.05$, significantly reduced as compared to control plasmid or wild-type SCC25 cells under hypoxia. 
A

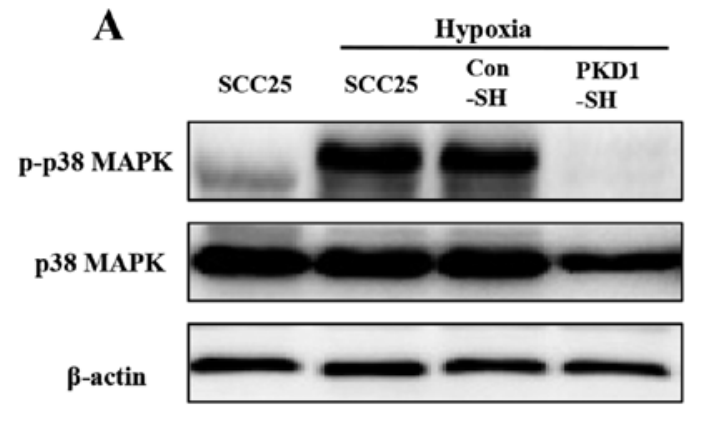

C

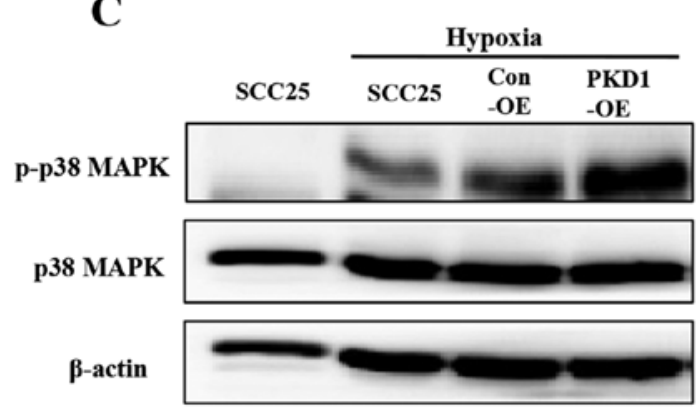

B

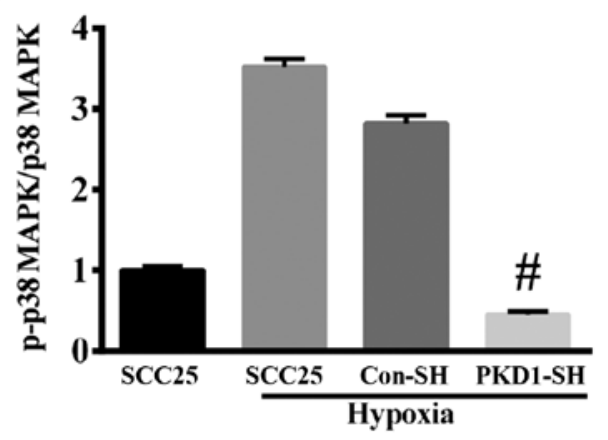

$\mathbf{D}$

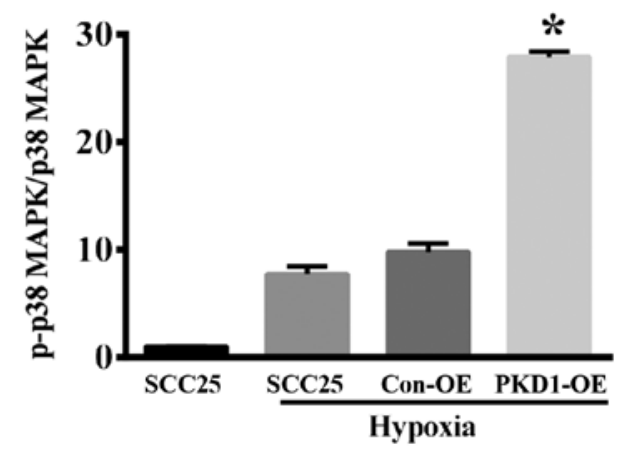

Figure 6. PKD1 promotes hypoxia-induced 338 MAPK expression and activation. PKD1-SH and PKD1-OE SCC25 cells were cultured in a humidified atmosphere of $5 \% \mathrm{CO}_{2}, 1 \% \mathrm{O}_{2}$ and $94 \% \mathrm{~N}_{2}$ at $37^{\circ} \mathrm{C}$. (A) The expression of p-p38 MAPK and p38 MAPK after PKD1 knockdown under hypoxia. (B) The ratio of p-p38 MAPK over p38 MAPK (PI) after PKD1 knockdown in SCC25 cells. (C) The expression of p-p38 MAPK and p38 MAPK after PKD1 overexpression under hypoxia. (D) The ratio of p-p38 MAPK over p38 MAPK (PI) after PKD1 overexpression in SCC25 cells. Figures are representative of three independent experiments. Data are presented as the mean $\pm \mathrm{SD}$. ${ }^{*} \mathrm{P}<0.05$, significantly increased as compared to the control plasmid or wild-type SCC25 cells under hypoxia. ${ }^{\#} \mathrm{P}<0.05$, significantly reduced as compared to the control plasmid or wild-type SCC25 cells under hypoxia.

GLUT1 and LDHA expression in SCC25 cancer cells (Fig. 5). When PKD1 was knocked down with PKD1-SH, the expression of GLUT1 and LDHA was significantly reduced as compared to wild-type SCC25 or control-SHSCC25 cells under hypoxia (Fig. 5A and B). Conversely, PKD1-overexpressing SCC25 cells had significantly increased expression of GLUT1 and LDHA under hypoxic conditions (Fig. 5C and D). These results indicate that PKD1 promotes glycolysis in oral squamous cancer cells by inducing the expression of glycolytic enzymes under hypoxic microenvironments.

Hypoxia induces the expression and activation of p-38 MAPK via PKD1. The p38 MAPKs are members of the MAPK family that are activated by a variety of environmental stresses and inflammatory cytokines. As shown in Fig. 6, hypoxia is an important environmental stress that activates p38 MAPK. SCC25 cells (wild-type SCC25, PKD1-SH and PKD1-OE) were cultured in a hypoxic environment for $24 \mathrm{~h}$, and the expression of p-p38 MAPK and p38 MAPK was analyzed via western blot analysis. The phosphorylation level of p38 MAPK was increased under hypoxia (Fig. 6A and C), whereas the expression level of total p38 MAPK was not statistically significantly different. The phosphorylation level of p38 MAPK and phosphorylated index (PI) of p-p38 MAPK/p38 MAPK were significantly decreased when SCC25 cells were transfected with PKD1-SH in a hypoxic environment for
$24 \mathrm{~h}$ (Fig. 6A and B). Moreover, the level of p-p38 MAPK and the PI of p-p38 MAPK/p38 MAPK were significantly increased when the PKD1-OE SCC25 cells were treated with hypoxia (Fig. 6C and D), compared with the expression level of total p38 MAPK.

\section{Discussion}

The tumor microenvironment is the 'resident niche' of cancer cells (1). A change in the tumor microenvironment can determine the survival or elimination of tumor cells (18). Targeting the tumor microenvironment has thus become an attractive strategy for cancer biotherapy (4).

Hypoxia and low $\mathrm{pH}$ are unique characteristics of the tumor microenvironment (6). When tumor growth outpaces angiogenesis within the tumor, the $\mathrm{O}_{2}$ level can be entirely suppressed within cancerous tissues $(31,32)$. Thus, tumor cells switch to a glycolytic metabolism to generate energy and secrete lactic acid, creating an acidic, hypoxic microenvironment $(12,17)$. Hypoxia induces the expression of HIF-1 $\alpha$, which controls the expression of hundreds of genes including tumor growth factors, metabolic enzymes and pro-metastatic factors that can promote the growth, metastasis and glucose metabolism of cancer cells, respectively. This unique microenvironment is also toxic to untransformed cells, inhibiting the antitumor immune response and increasing cancer cell drug resistance (33-36). As a result, cancer cells acquire an 
optimal 'resident microenvironment' and escape predation of the immune system $(37,38)$.

In the past decade, cancer metabolism has received a substantial amount of interest. Along with the progress of cancer genomics, proteomics and metabolomics, the connections between oncogenic signaling pathways and metabolic reprogramming in cancer are being increasingly recognized $(39,40)$.

Oncogenic signaling drives many of the metabolic responses of normal cells to growth-promoting signals (41). For example, AKT activation can increase glucose uptake, enhance activation and mitochondrial localization of hexokinase, and increase glycolytic flux. Both rapamycin complex 1 (mTORC1) and hypoxia-inducible factor (HIF) contribute to the increased expression and activation of glycolytic enzymes $(35,42)$. Additionally, MYC can promote the splicing of the pyruvate kinase gene PKM and enhance the expression of PKM2 (43).

PKD1, one of the PKD family members, is constitutively expressed in a variety of tumor cells (19). PKD1 signaling is involved in multiple bio-behaviors of cancer cells, including proliferation, apoptosis, migration, angiogenesis, oxidative stress response, autophagy and invasion (19). The expression and activation of PKD1 can be induced by multiple stimuli, such as growth factors, oxidative stress and chemokines $(19,44)$. In the present study, we found that hypoxia not only induced the expression of HIF-1 $\alpha$, but also induced the expression and activation (via phosphorylation) of PKD1 in SCC25 cells after culture for $6 \mathrm{~h}$ under hypoxic condition. Although the phosphorylated PKD1 decreased at $12 \mathrm{~h}$, it soon increased and arrived at a peak at $24 \mathrm{~h}$ (Fig. 1). Notably, although the phosphorylated PKD1 decreased at $6 \mathrm{~h}$, the expression of PKD1 and HIF-1 $\alpha$ still maintained a high level. This indicates that PKD1 is involved in the expression of HIF-1 $\alpha$ which is induced by hypoxia.

Both PKD1 and HIF-1 $\alpha$ are important regulators of cancer cell proliferation, differentiation, apoptosis and angiogenesis $(19,24)$. Furthermore, HIF-1 $\alpha$ is also an important regulator of cancer metabolism under hypoxic conditions (35). This study presents the role of PKD1 in the growth, apoptosis and metabolism under hypoxia in the most common type of human oral squamous cell carcinoma cells, SCC25. We cultured SCC 25 cells in $5 \% \mathrm{CO}_{2}, 1 \% \mathrm{O}_{2}$ and $94 \% \mathrm{~N}_{2}$ to simulate a hypoxic tumor microenvironment. When PKD1 expression was inhibited with shRNA, cell growth was significantly inhibited. In contrast, SCC25 cells expressed a high level of PKD1 under a hypoxic condition, thus, we transfected the exogenous PKD1 gene into the cells. Overexpression of PKD1 did not significantly affect the growth and apoptosis of SCC25 cells (Fig. 2). This indicates that PKD1 is a key regulator of cancer cell adaptation to the hypoxic microenvironment.

Hypoxia induces the expression of HIF-1 $\alpha$ to initiate the expression of tumor growth factors and glycolytic enzymes $(45,46)$, which provide growth signals and energy for tumor cells to adapt to and survive in a hypoxic microenvironment (45). After our previous finding that PKD1 promotes cancer cell growth and survival under hypoxia, we became interested in the role of PKD1 in cancer cell glycolysis under hypoxic conditions. We used RNA interference and enforced expression to suppressor overexpress PKD1 in oral squamous cell carcinoma cells ( $\mathrm{SCC} 25$ ), cultured in $5 \% \mathrm{CO}_{2}, 1 \% \mathrm{O}_{2}$ and
$94 \% \mathrm{~N}_{2}$ to simulate the hypoxic microenvironment. PKD1 knockdown with shRNA significantly decreased not only the expression of HIF-1 $\alpha$ (Fig. 3), but also the expression of GLUT1 and LDHA (Fig. 5). Similarly, when PKD1 was overexpressed in SCC25 cells, HIF-1 $\alpha$, GLUT1 and LDHA expression was significantly increased (Figs. 3 and 5). It is clear that HIF-1 $\alpha$ is a transcription regulator under hypoxia conditions. HIF-1 $\alpha$ regulates the metabolism of cancer cells by upregulating the expressions of glucose metabolism-related genes such as ENO1, pyruvate kinase 2 (PKM2), phosphoglycerate kinase 1 (PGK1), GLUT1 and LDHA in response to hypoxia (30). GLUT1 is the major carrier mediating glucose transport across mammalian cell membranes $(9,30)$. The function of LHDA is to convert pyruvate, one of the products of glucose metabolism, to lactate, which is then released into the extracellular milieu, lowering the $\mathrm{pH}$ of the tumor microenvironment $(6,47)$. Following the decreased expression of GLUT1 and LDHA after PKD1 inhibition, the ability of SCC25 cells to uptake glucose and release lactate was also decreased (Fig. 4). Conversely, when PKD1 was overexpressed in SCC25 cells, the ability of cancer cells to uptake glucose and release lactate was increased (Fig. 4). These results support the hypothesis that PKD1 is an important regulator of cancer cell glycolytic metabolism and the creation of an acidic microenvironment.

We next analyzed the expression and phosphorylation of p38 MAPK after PKD1 inhibition or overexpression under hypoxic conditions. p38 MAPK, which belongs to the MAPK superfamily, is a well-studied stress-activated kinase that transmits numerous extracellular signals and is involved in multiple cellular events $(48,49)$. p38 MAPK acts as a double-edged sword; in early stages, it acts as a tumor suppressor; however, it is required for cell survival in later stages of tumor progression (50). Hypoxia not only induces the expression of HIF-1 $\alpha$, but also increases the phosphorylation and activation of p38 MAPK in cancer cells (51). The activation of p38 MAPK signaling is necessary for HIF-1 $\alpha$ accumulation and nuclear translocation (52). In the present study, we observed that the knockdown and overexpression of PKD1 elevated the expression of p-p38 MAPK in order to further ascertain whether p-p38 MAPK is involved in the regulation of PKD1 in hypoxia. We found that PKD1 is associated with the activation of p38 MAPK signaling (Fig. 6). These results indicate that PKD1 plays an important role in hypoxia-induced expression and activation of HIF-1 $\alpha$ through p38 MAPK.

In conclusion, low $\mathrm{pH}$ and hypoxia are unique characteristics of the tumor microenvironment, which is a potential target for cancer treatment. Adaptation to this unique microenvironment is a basic requirement for cancer cells to survive. Our results have shown that PKD1 not only mediates the growth and apoptosis of cancer cells in the hypoxic environment, but also regulates the glycolytic metabolism of cancer cells by promoting glucose uptake and expression of HIF-1 $\alpha$ and glycolytic enzymes in a hypoxic environment. Inhibition of the expression and activation of PKD1 can significantly inhibit glycolytic metabolism of cancer calls as well as create an acidic tumor microenvironment. Hence, PKD1 may be considered as a potential target associated with microenvironment-directed tumor biotherapy. 


\section{Acknowledgements}

Not applicable.

\section{Funding}

The present study was financially supported by the National Natural Science Foundation of China (nos. 81372892, 81621062 and 81520108009), the 111 Project of MOE China (B14038), the Open Foundation from the State Key Laboratory of Oral Disease, Sichuan University (nos. SKLOD201601 and SKLOD2016OF01) and for the financial support, Sichuan Province Science and Technology Innovation Team Program (no. JCPT 2011-9).

\section{Availability of data and material}

The datasets used and/or analyzed during the current study are available from the corresponding author on reasonable request.

\section{Authors' contributions}

PZ designed the outline of the paper. JC, BMC and YPF conducted the experiments. XYL, QL and YD collected protein samples for western blot analysis. JC and BMC analyzed data. JC and PZ wrote the manuscript. JC and YPF prepared the figures. All authors read and approved the manuscript and agree to be accountable for all aspects of the research in ensuring that the accuracy or integrity of any part of the work are appropriately investigated and resolved.

\section{Ethics approval and consent to participate}

Not applicable.

\section{Patient consent for publication}

Not applicable.

\section{Competing interests}

The authors declare that they have no competing interests.

\section{References}

1. Anastasiou D: Tumour microenvironment factors shaping the cancer metabolism landscape. Br J Cancer 116: 277-286, 2017.

2. Alfarouk KO: Tumor metabolism, cancer cell transporters, and microenvironmental resistance. J Enzyme Inhib Med Chem 31: 859-866, 2016

3. Avantaggiati ML: Cancer metabolism as a therapeutic target: Finding the right target(s) in the context of tumor heterogeneity, evolution, and metabolic plasticity. Oncology 27: 474, 476-477, 2013.

4. Bahrami A, Khazaei M, Hassanian SM, ShahidSales S, JoudiMashhad M, Maftouh M, Jazayeri MH, Parizade MR, Ferns GA and Avan A: Targeting the tumor microenvironment as a potential therapeutic approach in colorectal cancer: Rational and progress. J Cell Physiol 233: 2928-2936, 2018.

5. Ackerman D and Simon MC: Hypoxia, lipids, and cancer: Surviving the harsh tumor microenvironment. Trends Cell Biol 24 472-478, 2014.

6. Brahimi-Horn MC and Pouysségur J: Hypoxia in cancer cell metabolism and $\mathrm{pH}$ regulation. Essays Biochem 43: 165-178, 2007.
7. Yamada K, Saito M, Matsuoka $\mathrm{H}$ and Inagaki N: A real-time method of imaging glucose uptake in single, living mammalian cells. Nat Protoc 2: 753-762, 2007.

8. Annibaldi A and Widmann C: Glucose metabolism in cancer cells. Curr Opin Clin Nutr Metab Care 13: 466-470, 2010.

9. Adekola K, Rosen ST and Shanmugam M: Glucose transporters in cancer metabolism. Curr Opin Oncol 24: 650-654, 2012.

10. Alfarouk KO, Verduzco D, Rauch C, Muddathir AK, Adil HH, Elhassan GO, Ibrahim ME, David Polo Orozco J, Cardone RA, Reshkin SJ, et al: Glycolysis, tumor metabolism, cancer growth and dissemination. A new $\mathrm{pH}$-based etiopathogenic perspective and therapeutic approach to an old cancer question. Oncoscience 1: 777-802, 2014.

11. Alfarouk KO, Verduzco D, Rauch C, Muddathir AK, Bashir AH, Elhassan GO, Ibrahim ME, Orozco JD, Cardone RA, Reshkin SJ, et al: Erratum: Glycolysis, tumor metabolism, cancer growth and dissemination. A new $\mathrm{pH}$-based etiopathogenic perspective and therapeutic approach to an old cancer question. Oncoscience 2: 317, 2014.

12. Frérart F, Sonveaux P, Rath G, Smoos A, Meqor A, Charlier N, Jordan BF, Saliez J, Noël A, Dessy C, et al: The acidic tumor microenvironment promotes the reconversion of nitrite into nitric oxide: Towards a new and safe radiosensitizing strategy. Clin Cancer Res 14: 2768-2774, 2008.

13. Doherty JR and Cleveland JL: Targeting lactate metabolism for cancer therapeutics. J Clin Invest 123: 3685-3692, 2013.

14. Justus CR, Dong L and Yang LV: Acidic tumor microenvironment and $\mathrm{pH}$-sensing $\mathrm{G}$ protein-coupled receptors. Front Physiol 4: 354, 2013.

15. Kato Y, Ozawa S, Miyamoto C, Maehata Y, Suzuki A, Maeda T and Baba Y: Acidic extracellular microenvironment and cancer. Cancer Cell Int 13: 89, 2013.

16. Koukourakis MI, Kakouratos C, Kalamida D, Bampali Z, Mavropoulou S, Sivridis E and Giatromanolaki A: Hypoxiainducible proteins HIF1 $\alpha$ and lactate dehydrogenase LDH5, key markers of anaerobic metabolism, relate with stem cell markers and poor post-radiotherapy outcome in bladder cancer. Int $\mathrm{J}$ Radiat Biol 92: 353-363, 2016.

17. Marchiq I and Pouysségur J: Hypoxia, cancer metabolism and the therapeutic benefit of targeting lactate $/ \mathrm{H}^{+}$symporters. $\mathrm{J}$ Mol Med (Berl) 94: 155-171, 2016.

18. Bailey KM, Wojtkowiak JW, Hashim AI and Gillies RJ: Targeting the metabolic microenvironment of tumors. Adv Pharmacol 65: 63-107, 2012.

19. LaValle CR, George KM, Sharlow ER, Lazo JS, Wipf P and Wang QJ: Protein kinase D as a potential new target for cancer therapy. Biochim Biophys Acta 1806: 183-192, 2010.

20. Mikhalap SV, Kovalevs'ka LM and Sydorenko SP: The role of PKD family protein kinases in the regulation of protein post-translational modification. Ukr Biokhim Zh 80: 16-24, 2008 (In Ukrainian).

21. Malhotra V and Campelo F: PKD regulates membrane fission to generate TGN to cell surface transport carriers. Cold Spring Harb Perspect Biol 3: a005280, 2011.

22. Eisenberg-Lerner A and Kimchi A: PKD at the crossroads of necrosis and autophagy. Autophagy 8: 433-434, 2012.

23. Liou GY and Storz P: Protein kinase D enzymes: Novel kinase targets in pancreatic cancer. Expert Rev Gastroenterol Hepatol 9: 1143-1146, 2015.

24. Mikhalap SV, Shabel'nyk MIu and Sydorenko SP: Protein kinases of PKD family as a potential object of translational research in oncology. Ukr Biokhim Zh (1999) 82: 18-32, 2010 (In Ukrainian).

25. No authors listed: Study reveals new drug target for PKD. Nephrol News Issues 30: 16, 2016.

26. Zhang T, Sell P, Braun U and Leitges M: PKD1 protein is involved in reactive oxygen species-mediated mitochondrial depolarization in cooperation with protein kinase $\mathrm{C} \delta$ (PKC $\delta$ ). J Biol Chem 290: 10472-10485, 2015.

27. Qin XJ, Gao ZG, Huan JL, Pan XF and Zhu L: Protein kinase D1 inhibits breast cancer cell invasion via regulating matrix metalloproteinase expression. Eur J Gynaecol Oncol 36: 690-693, 2015.

28. Ochi N, Tanasanvimon S, Matsuo Y, Tong Z, Sung B, Aggarwal BB, Sinnett-Smith J, Rozengurt E and Guha S: Protein kinase D1 promotes anchorage-independent growth, invasion, and angiogenesis by human pancreatic cancer cells. J Cell Physiol 226: 1074-1081, 2011.

29. Rozengurt E: Protein kinase D signaling: Multiple biological functions in health and disease. Physiology 26: 23-33, 2011. 
30. Jóźwiak P,Krześlak A,Bryś MandLipińskaA:Glucose-dependent glucose transporter 1 expression and its impact on viability of thyroid cancer cells. Oncol Rep 33: 913-920, 2015.

31. Teppo S, Sundquist E, Vered M, Holappa H, Parkkisenniemi J, Rinaldi T, Lehenkari P, Grenman R, Dayan D, Risteli J, et al: The hypoxic tumor microenvironment regulates invasion of aggressive oral carcinoma cells. Exp Cell Res 319: 376-389, 2013.

32. Pereira KM, Chaves FN, Viana TS, Carvalho FS, Costa FW, Alves AP and Sousa FB: Oxygen metabolism in oral cancer: HIF and GLUTs (Review). Oncol Lett 6: 311-316, 2013.

33. Marín-Hernández A, Gallardo-Pérez JC, Ralph SJ, RodríguezEnríquez S and Moreno-Sánchez R: HIF-lalpha modulates energy metabolism in cancer cells by inducing over-expression of specific glycolytic isoforms. Mini Rev Med Chem 9: 1084-1101, 2009.

34. Hay MP, Hicks KO and Wang J: Hypoxia-directed drug strategies to target the tumor microenvironment. Adv Exp Med Biol 772: 111-145, 2014.

35. Esteban MA and Maxwell PH: HIF, a missing link between metabolism and cancer. Nat Med 11: 1047-1048, 2005.

36. Leo C, Giaccia AJ and Denko NC: The hypoxic tumor microenvironment and gene expression. Semin Radiat Oncol 14: 207-214, 2004.

37. Labiano S, Palazon A and Melero I: Immune response regulation in the tumor microenvironment by hypoxia. Semin Oncol 42: 378-386, 2015.

38. Liu C, Gao S, Qu Z and Zhang L: Tumor microenvironment: Hypoxia and buffer capacity for immunotherapy. Med Hypotheses 69: 590-595, 2007.

39. No authors listed: Metabolism in cancer. JAMA 310: 2462, 2013.

40. Camarda R, Williams J and Goga A: In vivo reprogramming of cancer metabolism by MYC. Front Cell Dev Biol 5: 35, 2017.

41. Cantor JR and Sabatini DM: Cancer cell metabolism: One hallmark, many faces. Cancer Discov 2: 881-898, 2012.

42. Cornu M, Albert V and Hall MN: mTOR in aging, metabolism, and cancer. Curr Opin Genet Dev 23: 53-62, 2013.
43. Dang CV, Le A and Gao P: MYC-induced cancer cell energy metabolism and therapeutic opportunities. Clin Cancer Res 15: 6479-6483, 2009.

44. Liou GY, Storz P and Leitges M: A bright future for protein kinase D1 as a drug target to prevent or treat pancreatic cancer. Mol Cell Oncol 3: e1035477, 2015.

45. Finger EC and Giaccia AJ: Hypoxia, inflammation, and the tumor microenvironment in metastatic disease. Cancer Metastasis Rev 29: 285-293, 2010.

46. Airley RE and Mobasheri A: Hypoxic regulation of glucose transport, anaerobic metabolism and angiogenesis in cancer: Novel pathways and targets for anticancer therapeutics. Chemotherapy 53: 233-256, 2007.

47. Cui XG, Han ZT, He SH, Wu XD, Chen TR, Shao CH, Chen DL, Su N, Chen YM, Wang T, et al: HIF1/2 $\alpha$ mediates hypoxiainduced LDHA expression in human pancreatic cancer cells. Oncotarget 8: 24840-24852, 2017.

48. Matrone A, Grossi V, Chiacchiera F, Fina E, Cappellari M, Caringella AM, Di Naro E, Loverro G and Simone C: p38alpha is required for ovarian cancer cell metabolism and survival. Int $\mathbf{J}$ Gynecol Cancer 20: 203-211, 2010.

49. García-Cano J, Roche O, Cimas FJ, Pascual-Serra R, OrtegaMuelas M, Fernández-Aroca DM and Sánchez-Prieto R: p38 MAPK and Chemotherapy: We Always Need to Hear Both Sides of the Story. Front Cell Dev Biol 4: 69, 2016.

50. Brichkina A and Bulavin DV: Cancer suppression by systemic inactivation of p38 MAPK. Oncotarget 8: 14275-14276, 2017.

51. Koodie L, Ramakrishnan S and Roy S: Morphine suppresses tumor angiogenesis through a HIF-1alpha/p38 MAPK pathway. Am J Pathol 177: 984-997, 2010.

52. Yan L, Cao X, Zeng S, Li Z, Lian Z, Wang J, Lv F, Wang Y and Li Y: Associations of proteins relevant to MAPK signaling pathway (p38 MAPK-1, HIF-1 and HO-1) with coronary lesion characteristics and prognosis of peri-menopausal women. Lipids Health Dis 15: 187, 2016. 